\title{
Clinical value of quantitative analysis of ST slope during exercise
}

\author{
C. A. ASCOOP, C. A. DISTELBRINK, AND P. A. DE LANG \\ From the Department of Cardiology, St. Antonius Hospital, 2 fan van Scorelstraat, Utrecht; \\ and the Institute of Medical Physics TNO, 45 Da Costakade, Utrecht, The Netherlands
}

The diagnostic performance of automatic analysis of the exercise electrocardiogram in detecting ischaemic heart disease was studied in 147 patients with angiographically documented coronary disease. The results were compared with the results of visual analysis of the same recordings. Using a bicycle ergometer we tried to reach at least 90 per cent of the predicted maximal heart rate of the patient. Two bipolar thoracic leads (CM5, CC5) were used. In the visual analysis the criterion of the so-called ischaemic ST segment was applied. For the automatic analysis the population was divided into a learning group $(N=87)$ and a testing group $(N=60)$. In the learning group first critical values were computed for different $S T$ measurements that provided optimal separation between patients with (CAG. POS.) and without (CAG. NEG.) significant coronary stenoses as revealed by coronary arteriography. These critical values were kept unchanged when applied to the testing group. With respect to the visual method an increase of the sensitivity by 0.45 and 0.36 was obtained by the automatic analysis in the learning and testing group, respectively. The best separation between CAG. POS. and CAG. NEG. group was reached using a criterion consisting of a linear combination of the slope of the initial part of the ST segment and the ST depression; the sensitivity being 0.70 and 0.60, respectively, in the learning and testing group. Using a criterion based on the area between the baseline and the $S T$ segment (the $S X$ integral) these values were 0.42 and 0.49 , respectively. All specificities were kept to at least 0.90 .

Exercise electrocardiography plays an important role in the detection and assessment of ischaemic heart disease. It is a simple method for identifying coronary insufficiency especially in those patients in whom the history is atypical (Bartel et al., 1974). Furthermore because of its reproducibility, the test is frequently applied in the assessment of therapeutic and surgical treatment; the test also appears to have prognostic value (Ellestad and Wan, 1975). However, even if submaximal to maximal stress testing is used, some subjects fail to show electrocardiographic changes though their coronary arteriograms show significant stenoses (Ascoop et al., 1971). Various investigators studied new criteria which were introduced to increase the detection rate in patients with coronary disease. At the same time, however, the number of false positive cases rises because of observer variance (Punsar et al., 1968; McConahay et al., 1970). Measurements are performed more consistently by computer analysis (Sheffield et al., 1969; Ascopp et al., 1974; Yanowitz et al., 1974). It was the purpose of this study to assess various new criteria in exercise electrocardiography. Optimal critical values for these criteria were first derived in a 'learning population'; subsequently the diagnostic value of the criteria thus defined has been verified in a 'testing population'.

The QRS-T wave analysis was performed with the help of a digital computer; the exercise-induced electrocardiographic changes in each patient were compared with the findings from coronary angiography (CAG).

\section{Subjects and methods}

(1) One hundred and forty-seven consecutive patients, all men suspected of having ischaemic heart disease, were studied. The learning population consisted of 87 patients with an average age of $46( \pm 7)$ years; the testing population comprised 60 patients with an average age of $50( \pm 8)$ years. Previous examination in other hospitals from which the patients were referred yielded negative exercise electrocardiograms by visual analysis in most of them. 
The conditions for inclusion in this study were: (a) Normal repolarisation in the resting electrocardiogram; (b) no other cardiac disease present, except that which could be considered the result of ischaemic heart disease; (c) digitalis, beta-blocker, and antiarrhythmic agents were withheld two weeks before testing.

There were 6 patients in the learning group and 8 patients in the testing group with an infarct pattern. (2) A graded exercise test was performed in all patients imposing a multistage increasing workload by means of a calibrated bicycle ergometer. Starting at an external load of 60 Watt the load was increased every 3 minutes by $30 \mathrm{~W}$ until a target heart rate was reached of 90 per cent of the maximum heart rate predicted for the patient (Sheffield and Reeves, 1965), or until one of the 'stop' criteria was met (Ascoop et al., 1971).

(3) Recordings were made before the test (sitting), at the end of each exercise stage, and during the first 5 minutes of the recovery phase. The recorder is of the ink-jet type, frequency response linear up to $900 \mathrm{~Hz}$, and with a time constant of $0.01 \mathrm{~s}$; the paper speed was $25 \mathrm{~mm} / \mathrm{s}$. At the same time the signal was stored on magnetic tape using an FM recorder (Philips Analog 7, 9.5 cm/s).

(4) The electrocardiographic signals were derived from 2 bipolar thoracic leads with a common positive electrode at the position V5 of the standard electrocardiogram and the negative electrodes at the manubrium sterni (lead CM5) and at the position of V5R (lead CC5) (Blackburn et al., 1967).

(5) In the visual interpretation of the graphical recordings the standard criterion of ischaemic ST segment was applied and defined as follows: (a) a junction depression of at least 100 microvolts with respect to the ' $Q Q$ ' baseline; (b) followed by a horizontal or downsloping ST segment of at least $80 \mathrm{~ms}$ duration. A test was considered positive if 3 successive complexes showed an ischaemic ST segment at the stage of maximal load or during the first 3 minutes of the recovery phase, provided that the repolarisation wave at rest (sitting) was normal (Ascoop et al., 1971).

(6) Computer analysis was performed only on electrocardiograms made at the stage of maximal exercise load; these recordings comprise 30 seconds, about 20 seconds of which are averaged coherently after removal of aberrant complexes. By this method a single averaged complex is obtained; onset and end of its QRS wave are determined using a template method (Van Bemmel et al., 1973).

The baseline is defined at the level of the mean amplitude of the $10 \mathrm{~ms}$ sampling interval just before the QRS complex (sampling rate $300 \mathrm{~Hz}$ ). ST depressions, ST slopes, both up- and down- going and SX integrals are then computed, to establish new criteria.

\section{ST DEPRESSIONS}

These ST depressions measured at 1, 10, and $50 \mathrm{~ms}$ after the end of the QRS are called D0, D10 and D50, respectively.

\section{ST SLOPES (SL)}

These were calculated extending over three different intervals after the QRS complex: from 0 to $80 \mathrm{~ms}$ (SL 0-80), from 10 to $50 \mathrm{~ms}$ (SL 10-50), and from 50 to $70 \mathrm{~ms}$ (SL 50-70); (Fig. $1 \mathrm{C}, \mathrm{D}, \mathrm{E}$ ).

\section{SX INTEGRAL}

This was computed as the surface area between baseline and the negative part of the ST segment from junction to point $\mathrm{X}$, the first intersection between baseline and ST segment, but in no case further than the end point of the ST segment (Fig. 1A and B).

In addition the diagnotsic value of both ST depression and ST slope in combination are analysed: D0 with SL 0-80: (D0, SL 0-80) D10 with SL 10-50 (D10, SL 10-50) and D50 with SL 50-70 (D50, SL 50-70) (Fig. 1 C, D, E).

(7) The criteria described above were considered to predict the degree of coronary stenoses. The severity of coronary disease was determined independently by selective coronary arteriography according to Sones' technique, with additional craniocaudal projections (Sones and Shirey, 1962; Ludwig and Bruschke, 1973).

The published work (Bartel et al., 1974) and our own experience (Van Herpen et al., 1970) indicates that stenoses of at least 70 per cent of the lumen diameter in a major coronary branch are haemodynamically significant; the coronary arteriogram showing such lesions was classified as positive (CAG. POS.).

All other angiograms were classified negative (CAG. NEG.). The relation between prediction (exercise electrocardiogram) and reality (CAG) is presented in a yes-no association matrix. The number of correctly predicted CAG. POS. cases divided by the total number of positive cases is called the sensitivity of the test (correct positive fraction); the number of correctly predicted CAG. NEG. cases divided by the total number of CAG. NEG. cases is the specificity of the test (correct negative fraction). Critical values for the abovementioned criteria of ST depressions, ST slopes, and SX integral were determined in such a way that optimal separation between the CAG. POS. and the CAG. NEG. group was achieved in the learning population. 


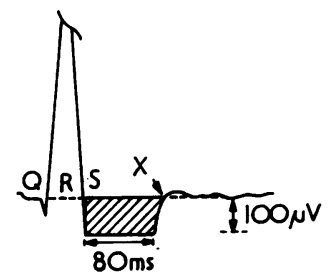

A
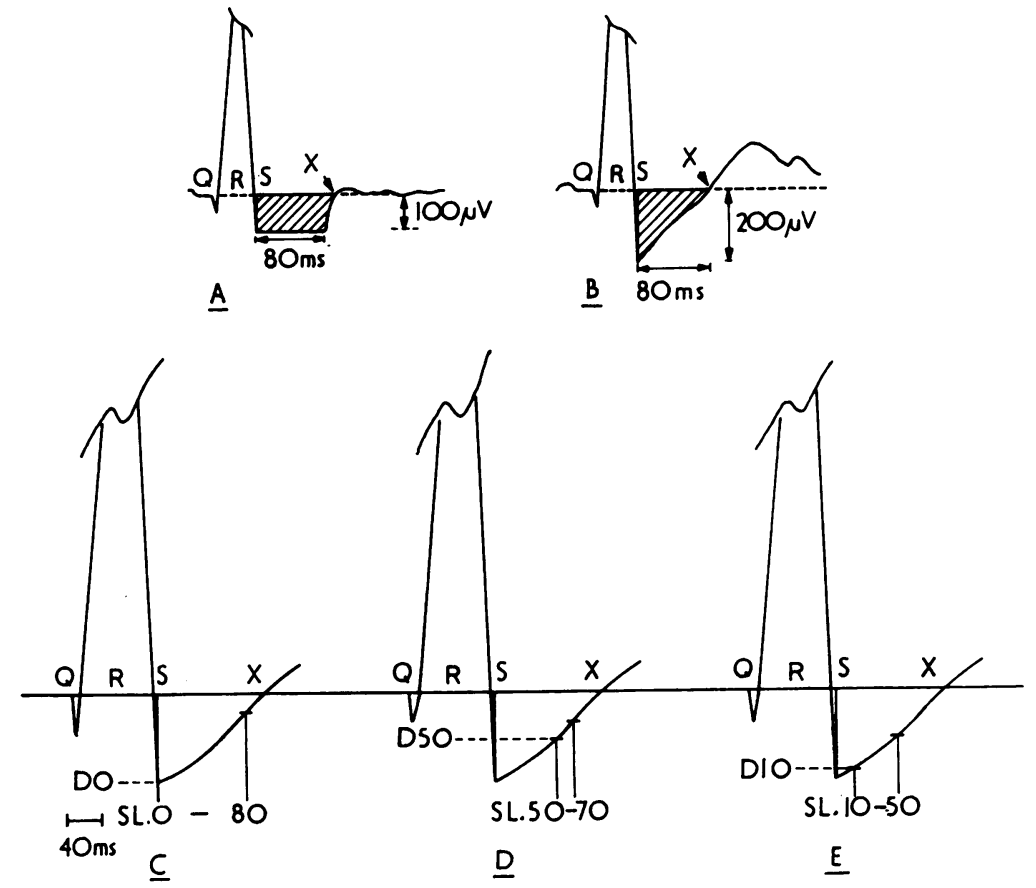

Fig. 1 Different measuring points on the $Q R S-T$ complex for calculating $S X$ integral ( $A$ and $B$ ), $S T$ depressions, and slopes $(C, D$ and $E)$.

We considered the separation optimal when a maximal sensitivity was achieved at a specificity of at least 0.9. These critical values of the criteria were subsequently kept unchanged when applied to the testing population.

\section{Results}

\section{(1) CORONARY ARTERIOGRAM}

Among the 87 patients of the learning population, 57 patients belonged to the CAG. POS. group (stenoses of at least 70 per cent in a major coronary branch). Of these 57 patients, 35 had an occlusion of at least one major branch. In this population 30 patients belonged to the CAG. NEG. group (stenoses of less than 70 per cent) and 26 of them had no coronary sclerosis at all.

In the testing population of 60 individuals, 39 patients were found to have significant coronary disease (CAG. POS.); 22 of them had an occlusion in at least one major coronary artery. Twenty-one patients belong to the CAG. NEG. group and in 13 of them no coronary sclerosis was detected.

(2) VISUAL ANALYSIS OF EXERCISE ELECTROCARDIOGRAM

Of the patients in the learning population, 14 showed ischaemic ST depressions, and all were
CAG. POS. The same analysis in the test group resulted in 11 positive responses, all in CAG. POS. cases. There was thus a specificity of 1.00 , but the sensitivity of this visual analysis in the learning and testing group was only 0.25 and 0.28 , respectively. Seventy per cent of the patients who showed a positive test were detected using lead CM5 only.

(3) COMPUTER ANALYSIS OF EXERCISE ELECTROCARDIOGRAM

$S T$ depressions

In the learning population the best results were obtained if the depression was measured $50 \mathrm{~ms}$ after the end of the QRS complex (D50) using a critical value of 35 microvolts in lead CC5 (Table 1). Sensitivities of 0.56 and 0.44 were then attained using leads CC5 and CM5, respectively. In the test population the sensitivity turned out to be 0.67 and 0.49 using leads $C C 5$ and CM5, respectively (Table 2).

\section{ST slope}

In the learning population the best predictions were obtained by the analysis of the slope of the early part of the ST segment (SL 10-50). The critical value for the SL $10-50$ in lead CC5 is 180 microvolts per $100 \mathrm{~ms}$ and for SL $0-80220$ microvolts per $100 \mathrm{~ms}$. Sensitivities of 0.65 and 0.54 were found for leads CC5 and CM5, respectively. Practically identical 
Table 1 Results in learning population $(N=87): 57$ patients with significant coronary disease (CAG. POS.) ; 30 patients without significant coronary disease (CAG. NEG.)

\begin{tabular}{|c|c|c|c|c|}
\hline Lead & Criterion & Critical values & Sensitivity & Specificity \\
\hline CM5 & 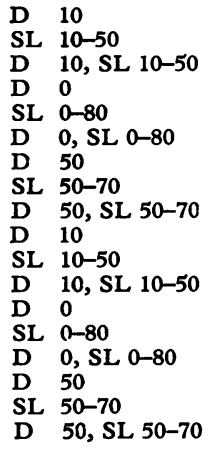 & $\begin{array}{l}D>154 \\
\text { SL } \leqq 180 \\
\text { SL }<0.75 \times D+122 \\
D \quad>178 \\
S L \leqq 220 \\
S L<0.87 \times D+122 \\
D \quad>35 \\
S L \leqq 200 \\
S L<1.59 \times D+234 \\
D \quad>200 \\
S L \leqq 190 \\
S L<0.30 \times D+165 \\
D \quad>220 \\
S L \leqq 235 \\
S L<0.31 \times D+184 \\
D \quad>90 \\
S L \leqq 239 \\
\text { SL }<0.54 \times D+204\end{array}$ & $\begin{array}{l}19 / 57=0.33 \\
37 / 57=0.65 \\
40 / 57=0.70 \\
16 / 57=0.28 \\
32 / 57=0.56 \\
34 / 57=0.60 \\
32 / 57=0.56 \\
20 / 57=0.35 \\
31 / 57=0.54 \\
13 / 57=0.23 \\
31 / 57=0.54 \\
34 / 57=0.60 \\
12 / 57=0.21 \\
35 / 57=0.61 \\
35 / 57=0.61 \\
25 / 57=0.44 \\
25 / 57=0.44 \\
27 / 57=0.47\end{array}$ & $\begin{array}{l}27 / 30=0.90 \\
27 / 30=0.90 \\
27 / 30=0.90 \\
27 / 30=0.90 \\
27 / 30=0.90 \\
27 / 30=0.90 \\
27 / 30=0.90 \\
27 / 30=0.90 \\
27 / 30=0.90 \\
28 / 30=0.93 \\
27 / 30=0.90 \\
27 / 30=0.90 \\
28 / 30=0.93 \\
27 / 30=0.90 \\
27 / 30=0.90 \\
27 / 30=0.90 \\
27 / 30=0.90 \\
27 / 30=0.90\end{array}$ \\
\hline
\end{tabular}

$\mathrm{Dt}, \mathrm{ST}$ depression in $\mu \mathrm{V} \mathrm{t} \mathrm{ms}$ after end of QRS.

$S L t_{1}-t_{2}$, ST slope in $\mu V / 100$ ms measured in interval $t_{1}-t_{2}$ ms after end of QRS.

results were obtained by measurements of SL $0-80$ which yielded sensitivities of 0.56 and 0.61 in leads CC5 and CM5, respectively (Table 1). In the testing population the SL 10-50 criterion yielded sensitivities of 0.54 and 0.46 in leads CC5 and CM5, respectively (Table 2 ).

\section{$S X$ integral}

Optimal separation in the learning population between the CAG. POS. and CAG. NEG. group was achieved at a critical value of $8 \mu \mathrm{V}$.s for lead CC5 and $10 \mu \mathrm{V}$.s for lead CM5. Application of this criterion resulted in a sensitivity of 0.42 and a specificity of 0.93 for the lead CC5 (Fig. 2). These values were 0.32 and 0.90 for the lead CM5. In the

Table 2 Results in test population $(N=60)$ : 39 patients with significant coronary disease (CAG. POS.) ; 21 patients without significant coronary disease (CAG. NEG.)

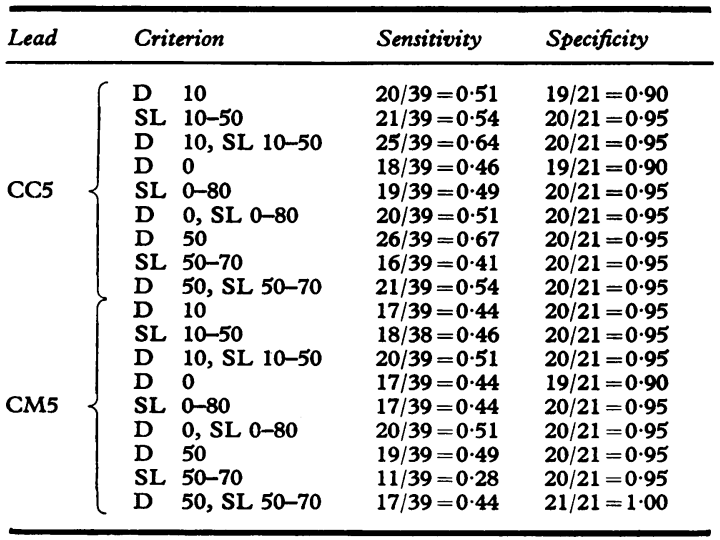

testing population the sensitivities were 0.49 using lead CC5 and 0.44 with lead CM5. The specificity was greater than 0.95 in both leads.

\section{Combinations of measurements}

Discrimination between CAG. POS. and CAG. NEG. was hereby obtained using a critical value computed through a linear combination of ST slope and ST depression. In the learning population, the best predictions yielded the combination D10, SL 10-50, using as discrimination function $S L=$ $0.75 \times D+122$ for $C C 5$ and $S L=0.30 \times D+165$ for CM5. This yielded a sensitivity of 0.70 and 0.60 , respectively. In the test population these values were 0.64 and 0.51 (Tables 1 and 2, Fig. 3 and 4).

\section{Discussion}

During the past decade the diagnostic performance of the exercise electrocardiogram has been increased

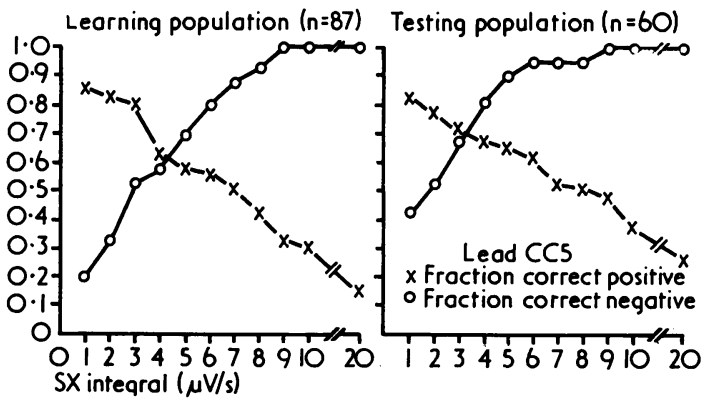

Fig. 2 Fraction correct positive (sensitivity) and fraction correct negative (specificity) at different values for the $S X$ integral in learning and test population. 


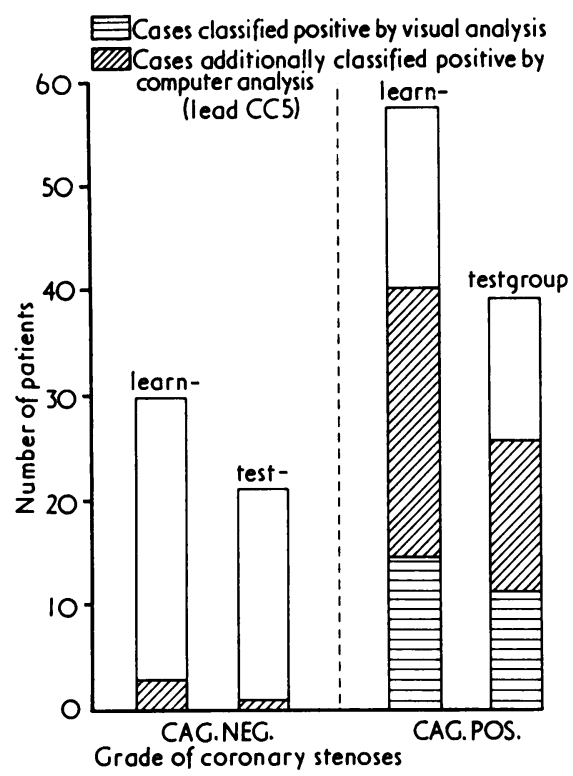

Fig. 3 Comparison of the diagnostic accuracy of visual and computer analysis. In the visual analysis use was made of the criterion of the ischaemic ST segment; in the automatic analysis a criterion was used based on a combination of ST depression and ST slope (D 10, SL 10-50); see methods.

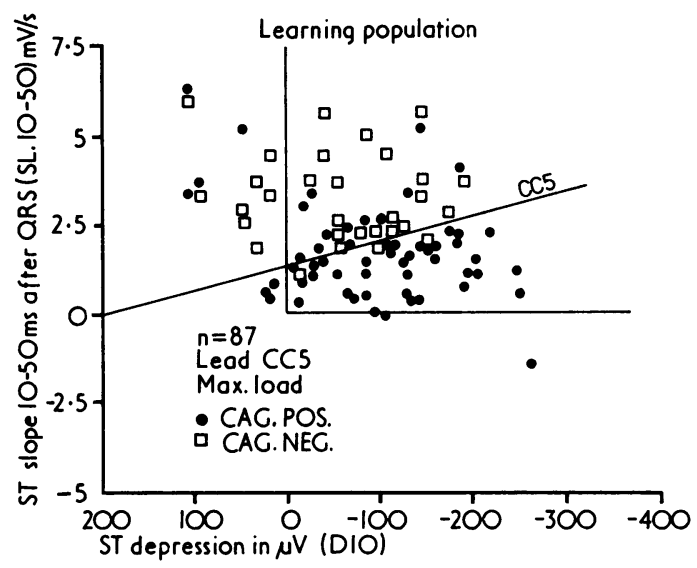

Fig. 4 Value of the $S T$ depression (D 10) and the $S T$ slope (SL 10-50) in the 87 patients in the learning population during the stage of maximal load. The function of the line for optimal classification is $S L=0.75 D+122$.

through the application of heavier work-loads (Mason et al., 1967; Ascoop et al., 1971). To augment further the sensitivity of the test different criteria have been proposed which represent detailed measurements of various characteristics of the ST-T segment. Application of these criteria or less rigid application of the standard criterion (see methods) often enlarges the number of false positive cases (Roman and Bellet, 1965).

The waveform of the ischaemic ST segment is determined on one hand by the consecutive ST vectors and on the other hand by the direction and strength of the lead (Burger et al., 1964). Study of vectorcardiograms during exercise shows that in exercise-induced ischaemia, the endpoints of the ST vectors of the initial part of the repolarisation, shift from left anterior and inferior to right posterior and superior; this shift appeared to be unrelated to the localisation of the coronary stenoses (Ascoop et al., 1974). Similar to the term 'ischaemic' ST segment used in scalar leads, we will use the term 'ischaemic ST vector' for ST vectors directed during exercise in the way described above. An ischaemic ST segment according to the conventional criterion can only occur if these ischaemic ST vectors exist for at least $80 \mathrm{~ms}$ and have a magnitude of at least 100 microvolts. As scalar leads such as CM5, CC5, and V5 have lead axes which coincide approximately with these ischaemic ST vectors, those leads are the most sensitive in exercise electrocardiography. Because of the selection criteria of our patients, application of the criterion of the ischaemic ST segment resulted in a low sensitivity in this study. However, it appears that ischaemic ST vectors with magnitudes less than 100 microvolts and correspondingly lower values in the ST segment already have a significant value in the detection of ischaemic heart disease. These measurements are reliable when performed automatically on averaged complexes. This study shows that the use of an ischaemic ST depression of 35 microvolts, $50 \mathrm{~ms}$ after QRS, results in sensitivities of 0.56 and 0.67 in the learning and testing population, respectively and in a specificity exceeding 0.90 in both groups. Use of ST depressions of 50 microvolts by visual analysis prove to have low specificity (Mason et al., 1967).

By automatic analysis diagnostic results are considerably better than by visual analysis if new criteria based upon ST slope, ST depression, and SX integral are used. The diagnostic accomplishments found here are, however, only applicable in male patients in whom the repolarisation wave at rest (sitting) is normal.

It appeared that 44 (lead CM5) to 49 per cent (lead CC5) of the CAG. POS. patients in our independent testing population could be detected by analysis of the SX integral. The critical value of the $S X$ integral in our material is $8 \mu \mathrm{V} . \mathrm{s}$, which is approximately the same value as Sheffield found in his study (Sheffield et al., 1969). In the evaluation 
of the ST slope, analysis of the initial part of the ST segment yields better results than analysis of the latter part.

A linear combination of ST slope (SL 10-50) and ST depression (D10) proves in this study to be the optimal criterion (see Fig. 3 and 4), yielding sensitivities of 0.70 and 0.64 per cent in learning and testing population, respectively. Good results are also reported by McHenry et al. (1972), who used measurements of the initial part of the ST segment; however, because of differences in criteria for patient selection comparison with our results is difficult (McHenry, Phillips, and Knoebel, 1972).

\section{CLINICAL IMPLICATIONS}

The fact that use of these new criteria, SX integral, D10, SL 10-50, and D50 both in learning and testing population yields a high sensitivity and specificity makes the application of this quantitative exercise electrocardiography attractive for clinical use. Previous studies show that use of orthogonal leads $(\mathrm{X}, \mathrm{Y}, \mathrm{Z})$ do not increase the diagnostic value of the exercise electrocardiogram (Distelbrink et al., 1976); it is advisable, therefore, to use leads CM5 and CC5. This has the additional advantage of reduction of the number of electrodes and consequently in reduction of sources of noise. If computer time is limited, recording of resting phase and of the stage of maximal load can be processed off-line. Continuous recordings with on-line processing increase the safety of exercise electrocardiography because the test can then be performed while monitoring the trend of the different measurements in comparison with the new exercise electrocardiographic criteria.

\section{References}

Ascoop, C. A., Distelbrink, C. A., De Lang, P. A., and Van Bemmel, J. H. (1974). Quantitative comparison of exercise vectorcardiograms and findings at selective coronary arteriography. Fournal of Electrocardiography, 7, 9.

Ascoop, C. A., Simoons, M. L., Egmond, W. G., and Bruschke, A. V. G. (1971). Exercise test, history, and serum lipid levels in patients with chest pain and normal electrocardiogram at rest: comparison to findings at coronary arteriography. American Heart fournal, 82, 609.

Bartel, A. G., Behar, V. S., Peter, R. H., Orgain, E. S., and Kong, Y. (1974). Graded exercise stress tests in angiographically documented coronary artery disease. Circulation, 49, 348.

Blackburn, H., Taylor, N. L., Okamoto, N., Rautaharju,
P.M., Mitchell, P., and Kerkhof, A. C. (1967). Standardization of exercise electrocardiogram. In Physical Activity and the Heart, p. 101. Ed. by M. J. Karvonen and A. J. Barry. Charles C. Thomas, Springfield, Illinois.

Burger, H. C., Van Brummelen, A. G. W., and Van Herpen, G. (1964). Compromise in vectorcardiography. II. Alterations of coefficients as a means of adapting one lead system to another. American Heart fournal, 64, 666.

Distelbrink, C. A., Ascoop, C. A., and De Lang, P. A. (1976). The diagnostic value of exercise electrocardiograms. In Advances in Cardiology, p. 529. Ed. by Hubert Abel. S. Karger, Basel.

Ellestad, M. H., and Wan, M. K. C. (1975). Predictive implications of stress testing. Circulation, 51, 363.

Ludwig, J. W., and Bruschke, A. V. (1973). Improvement of the diagnostic accuracy of the coronary arteriogram by unconventional projections. Circulation, Suppl. IV, 193, 4748.

McConahay, D. R., McCallister, B. D., Hallermann, F. J., and Smith, R. E. (1970). Comparative quantitative analysis of the electrocardiogram and the vectorcardiogram. Circulation, 42, 245.

McHenry, P. L., Phillips, J. F., and Knoebel, S. B. (1972) Correlation of computer-quantitated treadmill exercise electrocardiogram with arteriographic location of coronary artery disease. American fournal of Cardiology, 30, 747.

Mason, R. E., Likar, I. L., Biern, R. O., and Ross, R. S. (1967). Multiple-lead exercise electrocardiography. Circulation, 36, 517.

Punsar, S., Pyörölä, K., and Siltanen, P. (1968). Classification of electrocardiographic S-T segment changes in epidemiological studies of coronary heart disease. Annales Medicinae Internae Fenniae, 57, 53.

Roman, L., and Bellet, S. (1965). Significance of the QX/QT ratio and the $\mathrm{QT}$ ratio (QTr) in the exercise electrocardiogram. Circulation, 32, 435.

Sheffield, L. T., Holt, J. H., Lester, F. M., Conroy, D. V., and Reeves, T. J. (1969). On-line analysis of the exercise electrocardiogram. Circulation, 40, 935.

Sheffield, L. T., and Reeves, T. J. (1965). Graded exercise in the diagnosis of angina pectoris. Modern Concepts of Cardiovascular Disease, 34, 1.

Sones, F. M., and Shirey, E. K. (1962). Cine coronary arteriography. Modern Concepts of Cardiovascular Disease, 31, 735.

Van Bemmel, J. H., Talmon, J. L., Duisterhout, J. S., and Hengeveld, S. J. (1973). Template waveform recognition applied to ECG/VCG analysis. Computers and Biomedical Research, 6, 430.

Van Herpen, G., Bruschke, A. V. G., and Hansen, A. W. (1970). The correlation between coronary arteriogram and other diagnostic parameters. In Proceedings 11th International Vectorcardiography Symposium, p. 352. Ed. by I. Hoffman. North Holland, Amsterdam; Lippincott, Philadelphia.

Yanowitz, F., Froelicher, V. F., Jr., Keiser, N., and Lancaster, M. C. (1974). Quantitative exercise electrocardiography in the evaluation of patients with early coronary artery disease. Aerospace Medicine, 45, 443.

Requests for reprints to Dr. C. A. Ascoop, Department of Cardiology, St. Antonius Hospital, 2 Jan van Scorelstraat, Utrecht, The Netherlands. 